\title{
Intravitreal triamcinolone acetonide injection for macular edema due to central retinal vein occlusion persisting despite multiple intravitreal bevacizumab injections.
}

\author{
Rayan A Alshareef \\ Mid Atlantic Retina, Retina Service of Wills Eye Institute, Thomas Jefferson University \\ Sunir Garg \\ Mid Atlantic Retina, Retina Service of Wills Eye Institute, Thomas Jefferson University \\ Jason Hsu \\ Mid Atlantic Retina, Retina Service of Wills Eye Institute, Thomas Jefferson University \\ James Vander

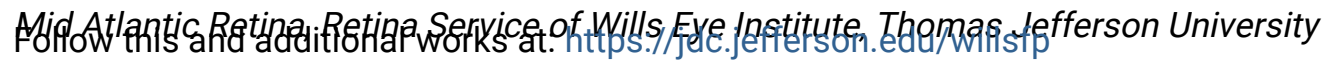 \\ IrpRardef the Ophthalmology Commons

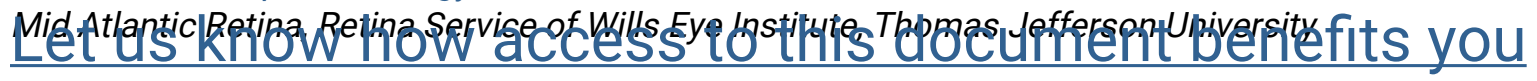

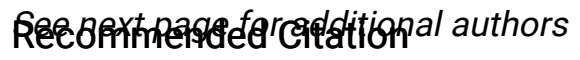

Alshareef, Rayan A; Garg, Sunir; Hsu, Jason; Vander, James; Park, Carl; and Spirn, Marc J, "Intravitreal triamcinolone acetonide injection for macular edema due to central retinal vein occlusion persisting despite multiple intravitreal bevacizumab injections." (2014). Wills Eye Hospital Papers. Paper 50.

https://jdc.jefferson.edu/willsfp/50

This Article is brought to you for free and open access by the Jefferson Digital Commons. The Jefferson Digital Commons is a service of Thomas Jefferson University's Center for Teaching and Learning (CTL). The Commons is a showcase for Jefferson books and journals, peer-reviewed scholarly publications, unique historical collections from the University archives, and teaching tools. The Jefferson Digital Commons allows researchers and interested readers anywhere in the world to learn about and keep up to date with Jefferson scholarship. This article has been accepted for inclusion in Wills Eye Hospital Papers by an authorized administrator of the Jefferson Digital Commons. For more information, please contact: JeffersonDigitalCommons@jefferson.edu. 


\section{Authors}

Rayan A Alshareef, Sunir Garg, Jason Hsu, James Vander, Carl Park, and Marc J Spirn 


\title{
Intravitreal Triamcinolone Acetonide Injection for Macular Edema Due to Central Retinal Vein Occlusion Persisting Despite Multiple Intravitreal Bevacizumab Injections
}

\author{
Rayan A. Alshareef, Sunir J. Garg, Jason Hsu, James Vander, Carl Park, and Marc J. Spirn
}

\begin{abstract}
Purpose: To evaluate the response to intravitreal triamcinolone acetonide for macular edema persisting or recurring despite multiple intravitreal bevacizumab (IVB) treatments for central retinal vein occlusion (CRVO). Methods: Retrospective interventional case series of 21 eyes with CRVO from 21 patients who were diagnosed with persistent or recurrent macular edema secondary to CRVO and treated with $0.1 \mathrm{~mL}(4 \mathrm{mg})$ intravitreal triamcinolone acetonide (IVTA) after initial treatment with 3 or more IVB injections. Anatomic and visual responses were the study primary outcomes.

Results: Mean logarithm of the minimum angle of resolution visual acuity was 1.19 (20/316) immediately before IVTA injection, and improved to $1.04(20 / 219) 1$ month after IVTA administration $(P=0.003)$. The mean central macular thickness on optical coherence tomography decreased from $533.4 \mu \mathrm{m}$ immediately before IVTA to $327.9 \mu \mathrm{m}$ after IVTA injection $(P<0.001)$. No cases of endophthalmitis, retinal detachment, or neovascularization were noted.

Conclusions: Intravitreal triamcinolone acetonide appears to improve vision and reduce persistent or recurrent macular edema secondary to CRVO despite multiple bevacizumab injections.
\end{abstract}

\section{Introduction}

$\mathbf{C}$ ENTRAL RETINAL VEIN OCCLUSION (CRVO) remains a major cause of significant visual morbidity due to retinal hemorrhage, macular ischemia, neovascular glaucoma, and macular edema. ${ }^{1}$ Although a number of therapeutic modalities have been used to treat CRVO-associated macular edema, intraocular injection of medications, most commonly intravitreal steroids (i.e., intravitreal triamcinolone acetonide [IVTA] and dexamethasone implant) and antivascular endothelial growth factor (VEGF) agents (i.e., pegaptanib, bevacizumab, ranibizumab, and aflibercept), are the most extensively studied and commonly used classes of mediations. VEGF is upregulated in patients with ischemic retinopathy, including CRVO. ${ }^{2,3}$ Both steroids and anti-VEGF agents reduce VEGF concentrations although through different mechanisms of action. ${ }^{4,5}$

The 2011 American Society of Retina Specialists' Preferences and Trends survey reported that many clinicians use anti-VEGF agents as first-line therapy for the treatment of CRVO, partly due to efficacy and partly due to reduced cataract formation and a lower incidence of increased in- traocular pressure compared with intraocular steroids. ${ }^{6}$ Several studies found visual benefits as well as reduction of macular edema from repeated use of intravitreal bevacizumab (IVB, Avastin; Genentech, Inc., South San Francisco, CA). ${ }^{7}$ More recently, a prospective randomized study of patients with CRVO-related macular edema found that between $46.2 \%$ and $47.7 \%$ of patients gain greater than or equal to 15 letters from baseline with monthly ranibizumab (Lucentis; Genentech, Inc.) compared with $16.9 \%$ of sham controls. ${ }^{8}$ Despite these encouraging results, $15 \%$ of patients treated with ranibizumab had no visual gain and $12 \%$ of patients actually lost vision despite therapy.

The dexamethasone implant (OZURDEX ${ }^{\circledR}$; Allergan, Inc.) and IVTA are considered the most commonly used intravitreal steroids. The dexamethasone implant is a potent corticosteroid that is approved by the United States Food and Drug Administration following results of 2 identical, randomized, prospective, multicenter, masked, sham-controlled, parallel group, phase 3 clinical trials that demonstrated significant therapeutic benefits compared with sham treatment for macular edema associated with BRVO or CRVO. ${ }^{9}$

Mid Atlantic Retina, The Retina Service of Wills Eye Institute, Thomas Jefferson University, Philadelphia, Pennsylvania. 
Triamcinolone acetonide is a corticosteroid that has been shown to improve vision and decrease macular edema in eyes with retinal vein occlusion. ${ }^{10,11} \mathrm{~A}$ randomized, shamcontrolled clinical trial demonstrated that for macular edema associated with CRVO, IVTA resulted in a significant improvement in both visual acuity as well as a decrease in macular edema. ${ }^{12,13}$ Despite a previous study with a small sample size that assessed the change in visual acuity and macular edema in patients with nonischemic CRVO who were previously unresponsive to IVB and who received IVTA, little is known about the efficacy of triamcinolone acetonide or the dexamethasone implant when administered in the setting of macular edema persisting or recurring following the administration of bevacizumab. ${ }^{14}$

Persistent or recurrent macular edema following antiVEGF therapy can be defined as a lack of retinal thickness reduction and/or a lack of improved visual acuity after repeated and continued dosing of anti-VEGF therapy.

Because the mechanisms of action of triamcinolone and anti-VEGF agents differ, some patients who do not respond adequately to anti-VEGF therapy may respond well to intraocular triamcinolone. We sought to evaluate whether patients with persistent macular edema due to a CRVO despite IVB injections respond to subsequent IVTA injection.

\section{Methods}

We retrospectively reviewed the charts of all patients initially treated with IVB for macular edema secondary to CRVO who subsequently received IVTA (Kenalog-40; Bristol-Myers Squibb, Princeton, NJ). The study protocol was approved by the Wills Eye Institute institutional review board and was conducted in accordance with the Declaration of Helsinki and all study patients signed a consent form before each injection. The following data were collected: age, gender, Snellen visual acuity measures at each visit, number, timing and type of intravitreal injections, assessment of pretreatment fluorescein angiography to determine the type of CRVO, optical coherence tomography (OCT) evaluation for central retinal thickness, and any adverse events. Patients were included if they received intravitreal triamcinolone acetonide for persistent or recurrent macular edema secondary to CRVO after at least 3 previous IVB injections. Exclusion criteria included less than 4 weeks of follow up after IVTA injection, previous focal or panretinal laser photocoagulation treatment, previous ranibizumab injection, and systemic steroid use. Changes in central macular thickness (CMT) and Snellen visual acuity were the primary endpoints.

Throughout the study period, all patients were scheduled for visits at 4- to 5-week intervals. Patient assessment and treatment was done within 5 days of the scheduled visit. Retreatment criteria included persistent or recurrent macular edema on clinical examination and/or vision loss. OCT was performed at the discretion of the treating physician. Reasons behind switching to IVTA included bevacizumab failure (persistent or recurrent macular edema and/or worsening vision), patient preference (to try an available alternative treatment method), and treating physician preference (perceived suboptimal response to IVB).

The 2 OCT machines used in this study were the Stratus OCT, a time domain OCT instrument, and the Cirrus HDOCT a spectral domain OCT instrument (both commercially available from Carl Zeiss Meditec, Inc., Dublin, CA).
Scanning with the Stratus OCT was performed by using the fast macular thickness map protocol, which acquires 6 evenly spaced 6-mm radial lines, consisting of 128 A-scans per line, intersecting at the fovea. Scanning with the Cirrus HD-OCT was performed with the macular cube $512 \times 128$ scan protocol where a $6 \times 6-\mathrm{mm}$ area on the retina is scanned with 128 horizontal lines, each consisting of 512 A-scans per line. CMT was obtained from the center subfield of the macular thickness map.

Statistical analyses were performed using Microsoft Excel (Microsoft Corporation, Redmond, WA). A 2-tailed student's $t$-test was used to compare visual acuity and OCT thickness results following both bevacizumab and triamcinolone acetonide treatments. The visual acuity measurements were converted to logarithm of the minimum angle of resolution $(\log$ MAR) units to facilitate statistical analysis.

\section{Results}

A total of 21 patients met the inclusion and exclusion criteria. There were a total of 12 males $(57 \%)$ and 9 females $(42.8 \%)$, with a mean age of 66.4 years (range 38-82 years). All patients had fluorescein angiography performed at the time of diagnosis. Nine eyes had ischemic CRVO, and 12 eyes had nonischemic CRVO. Patients received a mean of 4.1 bevacizumab injections (range $3-10$ ) before IVTA injection. Individual patient data can be found in Table 1 .

The mean visual acuity at initial diagnosis (before starting IVB therapy) was 1.21 (Snellen equivalent 20/330, range 20/ 70 to counting fingers at 2 feet). Initial bevacizumab treatment resulted in a mean $\log$ MAR visual acuity of 1.19 (Snellen equivalent 20/316, range 20/70 to counting fingers at 1 feet, $P=0.90$ ), and mean CMT on OCT was $533.4 \mu \mathrm{m}$ (range 300 $811 \mu \mathrm{m})$ before triamcinolone acetonide treatment.

On follow-up after triamcinolone injection, the mean $\log$ MAR visual acuity improved to 1.04 (Snellen equivalent was 20/219, range 20/50 to counting fingers at 3 feet, $P=0.003$ ), and mean macular thickness decreased significantly to $327.9 \mu \mathrm{m}$ (range $233-630 \mu \mathrm{m}, P<0.001$ ). Two patients did not show a decrease in CMT on OCT following IVTA, however, visual acuity improved in one of these patients. The other lost one line of vision compared with pre-IVTA measurements. On follow-up, 5 eyes received a second triamcinolone acetonide injection. One month after retreatment (at 2-month follow-up), all 5 eyes maintained stable visual acuity and displayed mild anatomic improvement. A subgroup analysis was performed based on the type of CRVO. Both nonischemic and ischemic eyes had a statistically significant reduction in CMT on follow-up [nonischemic $(P=0.003)$ and ischemic $(P=0.007)]$. Statistical significant improvement of visual acuity was noted in nonischemic eyes $(P=0.014)$, but not in eyes with ischemic CRVO $(P=0.062)$. Patients received a mean of 1.6 IVTA injections (range 1-4). Mean follow-up following IVTA was 13 weeks (range 12-15).

No significantly adverse events, including endophthalmitis, elevated intraocular pressure, or cataract formation, were noted in any patients during the follow-up period.

\section{Discussion}

Macular edema due to CRVO occurs due to increased venous pressure, loss of the integrity of the blood-retinal 
Table 1. Characteristics of Patients Before and After Switching to Triamcinolone Acetonide

\begin{tabular}{|c|c|c|c|c|c|c|c|}
\hline$I D$ & Age & $\begin{array}{c}\text { Type } \\
\text { of CRVO }\end{array}$ & $\begin{array}{c}\text { Prior } \\
\text { therapies }\end{array}$ & $\begin{array}{c}\text { Pre-IVTA } \\
\text { BCVA }\end{array}$ & $\begin{array}{c}\text { Post-IVTA } \\
\text { BCVA }\end{array}$ & $\begin{array}{c}\text { Pre-IVTA } \\
\text { CMT }\end{array}$ & $\begin{array}{c}\text { Post-IVTA } \\
\text { CMT }\end{array}$ \\
\hline 1 & 69 & I & B3 & CF 3 & CF 6 & 811 & 233 \\
\hline 2 & 65 & I & B5 & CF 3 & CF 4 & 345 & $412^{\mathrm{a}}$ \\
\hline 3 & 54 & I & B3 & $20 / 400$ & $20 / 300$ & 543 & 254 \\
\hline 4 & 65 & NI & B4 & $20 / 400$ & $20 / 100$ & 505 & 244 \\
\hline 5 & 68 & NI & B10 & $20 / 70$ & $20 / 50$ & 384 & 242 \\
\hline 6 & 67 & NI & B5 & CF 7 & $20 / 400$ & 537 & 237 \\
\hline 7 & 59 & NI & B3 & $20 / 200$ & $20 / 100$ & 305 & 280 \\
\hline 8 & 61 & I & B3 & $20 / 200$ & $20 / 200$ & 691 & $474^{\mathrm{a}}$ \\
\hline 9 & 69 & NI & B3 & $20 / 200$ & $20 / 300$ & 544 & $630^{\mathrm{a}}$ \\
\hline 10 & 80 & NI & B3 & CF 3 & $20 / 400$ & 455 & 320 \\
\hline 11 & 63 & I & B5 & $20 / 200$ & $20 / 200$ & 548 & 241 \\
\hline 12 & 65 & NI & B4 & $20 / 100$ & $20 / 70$ & 502 & 269 \\
\hline 13 & 82 & I & B3 & $20 / 200$ & $20 / 200$ & 492 & 396 \\
\hline 14 & 82 & NI & B4 & $20 / 80$ & $20 / 70$ & 539 & 260 \\
\hline 15 & 70 & NI & B3 & CF 6 & $20 / 400$ & 793 & 317 \\
\hline 16 & 38 & NI & B3 & CF 4 & CF 3 & 603 & $539^{\mathrm{a}}$ \\
\hline 17 & 76 & NI & B6 & CF 1 & CF 4 & 300 & 274 \\
\hline 18 & 65 & I & B4 & $20 / 200$ & $20 / 200$ & 758 & 493 \\
\hline 19 & 66 & I & B3 & $20 / 200$ & $20 / 200$ & 449 & $265^{\mathrm{a}}$ \\
\hline 20 & 65 & I & B6 & $20 / 80$ & $20 / 60$ & 315 & 239 \\
\hline 21 & 67 & NI & B4 & $20 / 100$ & $20 / 80$ & 780 & 268 \\
\hline Mean: & 66.4 years & & 4.18 & $\log \mathrm{MAR}$ & $\log \mathrm{MAR}$ & $533.4 \mu \mathrm{m}$ & $327.9 \mu \mathrm{m}^{\mathrm{c}}$ \\
\hline & & & injections & $1.19(20 / 316)$ & $1.04(20 / 214)^{\mathrm{b}}$ & & \\
\hline
\end{tabular}

Clinical features and outcomes for patients who had IVTA after refractory response to multiple intravitreal bevacizumab in CRVO. ${ }^{a}$ Retreatment.

${ }^{\mathrm{b}} P=0.003$.

${ }^{\mathrm{c}} P<0.001$.

The "Prior therapy" column lists prior therapies for macular edema before triamcinolone acetonide switch (B, bevacizumab; IVTA, intravitreal triamcinolone acetonide; $\mathrm{CF}$, counting fingers). Pretriamcinolone acetonide and post-triamcinolone acetonide OCT, and pretriamcinolone and post-triamcinolone acetonide BCVA columns list best-corrected visual acuities in Snellen notation. All thickness values were measured using OCT, and numbers listed are in microns, as defined in the Methods section.

BCVA, best-corrected visual acuity; CMT, central macular thickness; CRVO, central retinal vein occlusion; I, ischemic; logMAR, logarithm of the minimum angle of resolution; NI, non-ischemic; OCT, optical coherence tomography.

barrier, and damage to the vascular endothelial tight junction proteins. ${ }^{1}$ These dysregulations lead to increased VEGF levels, and increased VEGF expression appears to correlate with disease severity. ${ }^{2,3}$ Recently, VEGF inhibitors and steroids have been developed as a novel mechanism directed approach to treat macular edema secondary to CRVO. ${ }^{8} \mathrm{Ra}-$ nibizumab and bevacizumab are monoclonal antibodies that inhibit all isoforms of VEGF-A; they have been used to treat several conditions that cause macular edema and retinal ischemia. ${ }^{5,7}$ Results to date have been favorable and both ranibizumab and the off-label use of bevacizumab have resulted in improved visual acuity in several large clinical trials and case series. ${ }^{7,8,15-18}$ Although many patients have decreased macular edema and improved visual acuity with ongoing therapy, a significant number of patients have persistent macular edema despite anti-VEGF therapy.

The reasons for a persistent macular edema may be patient related or therapy regimen related. Patient-related factors include tachyphylaxis to the drug, drug resistance, increased rate of drug clearance, macular ischemia, or mechanical resistance such as a taut posterior hyaloid or an epiretinal membrane. ${ }^{19,20}$ Regimen-related reasons include treatment given too infrequently or stopped prematurely. Whereas anti-VEGF therapy reduces VEGF levels, processes such as dysregulation of endothelial tight junction proteins and the presence of inflammatory cytokines may persist resulting in persistent macular edema. ${ }^{21,22}$
Corticosteroids help mitigate many of the processes that contribute to the development of macular edema. The mode of action of triamcinolone acetonide not only downregulates VEGF expression but also decreases vascular permeability, stabilizes endothelial and retinal pigment epithelium tight junctions, decreases major histocompatibility class II expression, suppresses inflammatory cells, and inhibits the synthesis of cytokines and leukocyte movement. ${ }^{23,24}$ This retrospective study was performed to examine the effect of triamcinolone acetonide on macular edema refractory to multiple bevacizumab injections and highlights a method to decrease macular edema that persists despite multiple IVB treatments. In the current study, we noted a significant reduction in OCT thickness after triamcinolone acetonide, in patients who previously had a limited response to bevacizumab. Compared to pretriamcinolone macular thickness, the majority of eyes showed decreased macular edema (90.4\%) after triamcinolone injection. A similar proportion of patients $(90 \%)$ maintained or had improved visual acuity, although the acuity improvements were slight.

The design of this study included patients with refractory responses to bevacizumab. Based on visual acuity measurements of patients who were included in this study, it is likely that we have included patients with more severe (ischemic) disease. The reasons for lack of visual improvement are likely secondary to the substantial damage of the photoreceptor cells during the acute or chronic phase of CRVO or due to chronic macular 
edema. The larger trials such as Standard Care vs. Corticosteroid for Retinal Vein Occlusion (SCORE) Study and A Study of the Efficacy and Safety of Ranibizumab Injection in Patients with Macular Edema Secondary to Central Retinal Vein Occlusion (CRUISE) excluded patients with vision worse than 20/400. These patients likely had ischemic CRVO and were likely to have a less robust treatment response. Ischemic CRVO was not excluded in our study. This might be an explanation for the modest visual improvement seen in our series. Furthermore, in the CRUISE trial, many patients experienced rapid reduction in macular thickness and rapid improvement in visual acuity. $8,12,13$ Our study found both anatomic and visual benefits in a subgroup of patients who had ischemic disease.

This study is limited by its nonrandomized, noncomparative and retrospective nature. The average number of anti-VEGF injections (4.1) and triamcinolone acetonide injections (1.6) was small compared with the CRUISE and SCORE studies, respectively, and additional injections may have enabled greater, although still modest, visual improvement. No significant complications developed in our patients after treatment with bevacizumab or triamcinolone acetonide, however, cataract formation and an increase in intraocular pressure may have occurred with a longer follow-up.

\section{Conclusions}

This study evaluated the anatomical and visual effect of triamcinolone acetonide on eyes previously treated with IVB. The majority of patients experienced an improvement in visual acuity, IVTA provided stability of visual acuity in other patients.

In summary, this study suggests that switching to IVTA injection may be an effective treatment for patients with persistent or recurrent macular edema secondary to CRVO following multiple treatments with bevacizumab. Further trials may help understand which patients may benefit most from this treatment modality.

\section{Author Disclosure Statement}

There are no commercial associations that create a conflict of interest in connection with the submitted material.

\section{References}

1. Central Vein Occlusion Study Group. Natural history and clinical management of central retinal vein occlusion. Arch. Ophthalmol. 115:486-491, 1997.

2. Boyd, S.R., Zachary, I., Chakvarthy, U., et al. Correlation of increased vascular endothelial growth factor with neovascularization and permeability in ischemic central vein occlusion. Arch. Ophthalmol. 120:1644-1650, 2002.

3. Aiello, L.P., Avery, R.L., Arrigg, P.G., et al. Vascular endothelial growth factor in ocular fluid of patients with diabetic retinopathy and other retinal disorders. N. Engl. J. Med. 331:1480-1487, 1994.

4. Sears, J.E., and Hoppe, G. Triamcinolone acetonide destabilizes VEGF mRNA in Muller cells under continuous cobalt stimulation. Invest. Ophthalmol. Vis. Sci. 46:4336-4341, 2005.

5. Qian, J., Lu, Q., Tao, Y., and Jiang, Y.R. Vascular endothelial growth factor in aqueous humor before and after intravitreal injection of bevacizumab in eyes with diabetic retinopathy. Retina. 31:161-168, 2011.
6. American Society of Retina Specialists Annual Preferences and Trends Survey, 2010. www.asrs.org; access for members only.

7. Hsu, J., Kaiser, R.S., Sivalingam, A., Abraham, P., et al. Intravitreal bevacizumab (avastin) in central retinal vein occlusion. Retina. 27:1013-1019, 2007.

8. Brown, D.M., Campochiaro, P.A., Singh, R.P., et al. Ranibizumab for macular edema following central retinal vein occlusion: six-month primary end point results of a phase III study. Ophthalmology. 117:1124-1133, 2010.

9. Haller, J.A., Bandello, F., Belfort, R., Jr., et al. Randomized, sham-controlled trial of dexamethasone intravitreal implant in patients with macular edema due to retinal vein occlusion. Ophthalmology. 117:1134-1146, e1133, 2010.

10. Ramezani, A., Entezari, M., Moradian, S., et al. Intravitreal triamcinolone for acute central retinal vein occlusion: a randomized clinical trial. Graefes Arch. Clin. Exp. Ophthalmol. 244:1601-1606, 2006.

11. Ip, M.S., Gottlieb, J.L., Kahana, A., et al. Intravitreal triamcinolone for the treatment of macular edema associated with central retinal vein occlusion. Arch. Ophthalmol. 122:1131-1136, 2004.

12. Ip, M.S., Scott, I.U., VanVeldhuisen, P.C., Oden, N.L., Blodi, B.A., Fisher, M., Singerman, L.J., Tolentino, M., Chan, C.K., and Gonzalez, V.H. A randomized trial comparing the efficacy and safety of intravitreal triamcinolone with observation to treat vision loss associated with macular edema secondary to central retinal vein occlusion: the Standard Care vs Corticosteroid for Retinal Vein Occlusion (SCORE) study report 5. Arch. Ophthalmol. 127:1101-1114, 2009. Erratum in: Arch. Ophthalmol. 127:1648, 2009.

13. Domalpally, A., Blodi, B.A., Scott, I.U., Ip, M.S., Oden, N.L., Lauer, A.K., and VanVeldhuisen, P.C. The Standard Care vs Corticosteroid for Retinal Vein Occlusion (SCORE) study system for evaluation of optical coherence tomograms: SCORE study report 4. Arch. Ophthalmol. 127:1461-1467, 2009.

14. Jonas, J.B., Libondi, T., Schlichtenbrede, F., and Schmidbauer, M. Intravitreal triamcinolone after intravitreal bevacizumab for retinal vein occlusions. Acta Ophthalmol. 88:e24-e25, 2010.

15. Rosenfeld, P.J., Fung, A.E., and Puliafito, C.A. Optical coherence tomography findings after an intravitreal injection of bevacizumab (Avastin) for macular edema from central retinal vein occlusion. Ophthalmic Surg. Lasers Imaging. 36:336-339, 2005.

16. Iturralde, D., Spaide, R.F., Meyerle, C.B., et al. Intravitreal bevacizumab (Avastin) treatment of macular edema in central retinal vein occlusion: a short-term study. Retina. 26:279-284, 2006.

17. Costa, R.A., Jorge, R., Calucci, D., et al. Intravitreal bevacizumab (Avastin) for central and hemicentral retinal vein occlusions: IBeVO Study. Retina. 27:141-149, 2007.

18. Beutel, J., Ziemssen, F., Luke, M., et al. Intravitreal bevacizumab treatment of macular edema in central retinal vein occlusion: one-year results. Int. Ophthalmol. 30:1522, 2010.

19. Schaal, S., Kaplan, H.J., and Tezel, T.H. Is there tachyphylaxis to intravitreal anti-vascular endothelial growth factor pharmacotherapy in age-related macular degeneration? Ophthalmology. 115:2199-2205, 2008.

20. Forooghian, F., Cukras, C., Meyerle, C.B., Chew, E.Y., and Wong, W.T. Tachyphylaxis after intravitreal bevacizumab for exudative age-related macular degeneration. Retina. 29:723-731, 2009. 
21. Fulya Ilhan, Ulku Celiker, Ahmet Godekmerdan, Emrah Kan, et al. The Antiphospholipid Antibody Syndrome Research in Patients with Retinal Venous Occlusion. Arch. Med. Res. 36:372-375, 2005.

22. Green, W.R., Chan, C.C., and Hutchins, G.M. Central retinal vein occlusion: a prospective histopathologic study of 29 eyes in 28 cases. Trans. Am. Ophthalmol. Soc. 371-422, 1981.

23. Leopold, I.H. Nonsteroidal and steroidal anti-inflammatory agents. In: Sears, M.L., Tarkkanen, A., eds. Surgical Pharmacology of the Eye. New York: Raven Press, 1985; p. 83133.

24. Penfold, P.L., Wong, J.G., Gyory, J., and Billson, F.A. Effects of triamcinolone acetonide on microglial morphology and quantitative expression of MHC-II in exuda- tive age-related macular degeneration. Clin. Experiment. Ophthalmol. 29:188-192, 2001.

Received: October 11, 2013

Accepted: March 9, 2014

Address correspondence to:

Dr. Marc J. Spirn

Mid Atlantic Retina

The Retina Service of Wills Eye Institute

Thomas Jefferson University

840 Walnut Street, Suite 1020

Philadelphia, PA 19107

E-mail: spirnm01@yahoo.com 